\title{
Decision Problems for Deterministic Pushdown Automata on Infinite Words
}

\author{
Christof Löding \\ Lehrstuhl Informatik 7 \\ RWTH Aachen University \\ Germany \\ loeding@cs.rwth-aachen.de
}

\begin{abstract}
The article surveys some decidability results for DPDAs on infinite words ( $\omega$-DPDA). We summarize some recent results on the decidability of the regularity and the equivalence problem for the class of weak $\omega$-DPDAs. Furthermore, we present some new results on the parity index problem for $\omega$ DPDAs. For the specification of a parity condition, the states of the omega-DPDA are assigned priorities (natural numbers), and a run is accepting if the highest priority that appears infinitely often during a run is even. The basic simplification question asks whether one can determine the minimal number of priorities that are needed to accept the language of a given $\omega$-DPDA. We provide some decidability results on variations of this question for some classes of $\omega$-DPDAs.
\end{abstract}

\section{Introduction}

Finite automata, which are used as a tool in many areas of computer science, have good closure and algorithmic properties. For example, language equivalence and inclusion are decidable (see [9]), and for many subclasses of the regular languages it is decidable whether a given automaton accepts a language inside this subclass (see [19] for some results of this kind). In contrast to that, the situation for pushdown automata is much more difficult. For nondeterministic pushdown automata, many problems like language equivalence and inclusion are undecidable (see [9]), and it is undecidable whether a given nondeterministic pushdown automaton accepts a regular language. The class of languages accepted by deterministic pushdown automata forms a strict subclass of the context-free languages. While inclusion remains undecidable for this subclass, a deep result from [15] shows the decidability of the equivalence problem. Furthermore, the regularity problem for deterministic pushdown automata is also decidable [17, 20].

While automata on finite words are a very useful model, some applications, in particular in verification by model checking (see [2]), require extensions of these models to infinite words. Although the theory of finite automata on infinite words (called $\omega$-automata in the following) usually requires more complex constructions because of the more complex acceptance conditions, many of the good properties of finite automata on finite words are preserved (see [13] for an overview). Pushdown automata on infinite words (pushdown $\omega$-automata) have been studied because of their ability to model executions of nonterminating recursive programs. In [6] efficient algorithms for checking emptiness of Büchi pushdown automata are developed (a Büchi automaton accepts an infinite input word if it visits an accepting state infinitely often during its run). Besides these results, the algorithmic theory of pushdown $\omega$-automata has not been investigated very much. For example, in [5] the decidability of the regularity problem for deterministic pushdown $\omega$-automata has been posed as an open question and to our knowledge no answer to this question is known. Furthermore, it is unknown whether the equivalence of deterministic pushdown $\omega$-automata is decidable.

Z. Ésik and Z. Fülöp (Eds.): Automata and Formal Languages 2014 (AFL 2014) EPTCS 151, 2014, pp. 55-73, doi 10.4204/EPTCS.151.4 (c) C. Löding

This work is licensed under the Creative Commons Attribution License. 
The first part of this article summarizes some recent partial results on the regularity and equivalence problem for deterministic pushdown $\omega$-automata from [12].

In the second part we consider decision problems concerning the acceptance condition of the automata. One of the standard acceptance conditions of $\omega$-automata is the parity condition (see [8] for an overview of possible acceptance conditions). Such a condition is specified by assigning priorities (natural numbers) to the states of the automaton, using even priorities for "good" states and odd priorities for the "bad" states. A run is accepting if among the states that occur infinitely often the highest priority is even. For deterministic automata (independent of the precise automaton model), one can show that more languages can be accepted if more priorities are used. So the number of priorities required for accepting a language is a measure for the complexity of the language. A natural decision problem arising from that, is the question of determining for a given deterministic parity automaton the smallest number of priorities that are needed for accepting the language of the automaton. This referred to as the parity index problem.

For finite deterministic parity automata, the minimal number of priorities required for accepting the language can be computed in polynomial time, and a corresponding automaton can be constructed by simply reassigning priorities in the allowed range to the states of the given automaton [4]. For deterministic pushdown parity automata it was shown in [10] that it is decidable whether a given automaton is equivalent to a deterministic pushdown Büchi automaton. We present here the general result that the parity index problem for deterministic pushdown parity automata is decidable. The method is based on parity games on pushdown graphs and has already been described in the $\mathrm{PhD}$ thesis [14].

We further consider a model of deterministic pushdown automata in which the types of the action on the pushdown store are determined by the input symbols, called visibly pushdown automata (VPA) [1]. In these automata, the input alphabet is partitioned into three sets of symbols, referred to as call, return, and internal symbols. On reading a call, the pushdown automaton has to add a symbol to the stack, on reading a return, it has to remove a symbol from the stack, and on reading an internal, it does not alter the stack. It turns out that, for a fixed partition of the input alphabet, this class of automata has good closure and algorithmic properties [1]. On finite words it is even possible to determinize such VPAs. However, it turns out that Büchi VPAs cannot, in general, be transformed into equivalent deterministic Muller or parity VPAs [1]. To resolve this problem, in [11] a variation of the parity condition has been proposed, referred to as stair parity condition. It is defined as a standard parity condition, however, it is not evaluated on the sequence of all states but only on the sequence of states that occur on steps of the run. A step is a configuration in the run such that no later configuration has a smaller stack height. In [11] it is shown that each nondeterministic Büchi VPA can be transformed into an equivalent deterministic stair parity VPA. We prove here that the stair parity index problem for deterministic VPAs can be solved in polynomial time. We also consider the question whether a given stair parity VPA is equivalent to a parity VPA (with a standard parity condition instead of a stair condition). For the particular case of stair Büchi VPAs we show that this problem is decidable.

The remainder of this paper is structured as follows. In Section 2 we introduce some basic terminology and definitions. In Section 3 we consider the regularity and equivalence problem for $\omega$-DPDAs. Section 4 is about the parity index of parity DPDAs and stair parity DVPAs. In Section 5 we show how to decide whether the stair condition is needed for accepting the language of a given stair Büchi DVPAs. In Section 6 we give a short conclusion. 


\section{Preliminaries}

We denote the set of natural numbers (including 0) by $\mathbb{N}$. For a set $S$ we denote its cardinality by $|S|$. Let $A$ be an alphabet, i.e., a finite set of symbols, then $A^{*}$ is the set of finite words over $A$, and $A^{\omega}$ the set of $\omega$-words over $A$, i.e., infinite sequences of $A$ symbols indexed by the natural numbers. The subsets of $A^{*}$ are called languages, and subsets of $A^{\omega}$ are called $\omega$-languages. The length of a finite word $w \in A^{*}$ is denoted by $|w|$, and the empty word is $\varepsilon$. We assume the reader to be familiar with regular languages, i.e., the languages specified by regular expressions or equivalently by finite state automata (see, for example, [9] for basics on regular languages).

We are mainly concerned with deterministic pushdown automata in this work. We first define pushdown machines, which are pushdown automata without acceptance condition. We then obtain pushdown automata by adding an acceptance condition.

A deterministic pushdown machine $\mathscr{M}=\left(Q, A, \Gamma, \delta, q_{0}, \perp\right)$ consists of

- a finite state set $Q$ and initial state $q_{0} \in Q$,

- a finite input alphabet $A$ (we abbreviate $A_{\varepsilon}=A \cup\{\varepsilon\}$ ),

- a finite stack alphabet $\Gamma$ and initial stack symbol $\perp \notin \Gamma$ (let $\left.\Gamma_{\perp}=\Gamma \cup\{\perp\}\right)$,

- a partial transition function $\delta: Q \times \Gamma_{\perp} \times A_{\varepsilon} \rightarrow Q \times \Gamma_{\perp}^{*}$ such that for each $p \in Q$ and $A \in \Gamma_{\perp}$ :

- $\delta(p, Z, a)$ is defined for all $a \in A$ and $\delta(p, Z, \varepsilon)$ is undefined, or the other way round.

- For each transition $\delta(p, Z, a)=(q, W)$ with $a \in A_{\varepsilon}$ the bottom symbol $\perp$ stays at the bottom of the stack and only there, i.e., $W \in \Gamma^{*} \perp$ if $Z=\perp$ and $W \in \Gamma^{*}$ if $Z \neq \perp$.

The set of configurations of $\mathscr{M}$ is $Q \Gamma^{*} \perp$ where $q_{0} \perp$ is the initial configuration. The stack consisting only of $\perp$ is called the empty stack. A configuration $q \sigma$ is also written $(q, \sigma)$. For a given input word $w \in A^{*}$ or $w \in A^{\omega}$, a finite resp. infinite sequence $q_{0} \sigma_{0}, q_{1} \sigma_{1}, \ldots$ of configurations with $q_{0} \sigma_{0}=q_{0} \perp$ is a run of $w$ on $\mathscr{M}$ if there are $a_{i} \in A_{\varepsilon}$ with $w=a_{1} a_{2} \cdots$ and $\delta\left(q_{i}, Z, a_{i+1}\right)=\left(q_{i+1}, U\right)$ is such that $\sigma_{i}=Z V$ and $\sigma_{i+1}=U V$ for some stack suffix $V \in \Gamma_{\perp}^{*}$.

For finite words, we consider the model of a deterministic pushdown automaton (DPDA) $\mathscr{A}=$ $(\mathscr{M}, F)$ consisting of a deterministic pushdown machine $\mathscr{M}=\left(Q, A, \Gamma, \delta, q_{0}, \perp\right)$ and a set of final states $F \subseteq Q$. It accepts a word $w \in A^{*}$ if $w$ induces a run ending in a final state. These words form the language $L_{*}(\mathscr{A}) \subseteq A^{*}$. For $\omega$-words, we consider two types of acceptance conditions, namely Büchi and parity conditions. A Büchi DPDA $\mathscr{A}=(\mathscr{M}, F)$ is specified in the same way as a DPDA on finite words. The $\omega$-language $L_{\omega}(\mathscr{A})$ defined by $\mathscr{A}$ is the set of all $\omega$-words $w$ for which the run of $\mathscr{A}$ on $w$ contains a state from $F$ at infinitely many positions.

For a parity DPDA, the acceptance condition is specified by a function $\Omega: Q \rightarrow \mathbb{N}$, which assigns a number to each state, which is referred to as its priority. A run is accepting if the highest priority that occurs infinitely often is even. Note that Büchi conditions can be specified as parity conditions by assigning priority 2 to states in $F$ and priority 1 to states outside $F$.

In Section 3 we consider the class of weak DPDAs. These are parity DPDAs, in which the transitions can never lead from one state $q$ to another state $q^{\prime}$ with a smaller priority. Hence, in a run of a weak DPDA the sequence of priorities is monotonically increasing, which implies that the sequence is ultimately constant. It follows that each weak DPDA is equivalent to the Büchi DPDA that uses the set of states with even priority as set of final states. We therefore also use term weak Büchi DPDAs to emphasize that it is a subclass of Büchi DPDAs.

In general, we refer to DPDAs on infinite words as $\omega$-DPDAs if we do not explicitly specify the type of acceptance. For simplicity, we assume that infinite sequences of $\varepsilon$-transitions are not possible 
in $\omega$-DPDAs. Such sequences can be eliminated by redirecting certain $\varepsilon$-transitions into corresponding sink states (the acceptance status of such a state would depend on the exact semantics one uses for runs that end in an infinite $\varepsilon$-sequence). It is sufficient to compute the pairs $(q, Z)$ of states $q$ and top stack symbols $Z$ such that there is a run of $\varepsilon$-transitions leading from $q Z \perp$ to some configuration of the form $q Z W Z \perp$, such that the $Z$ at the bottom of the stack is never removed during this run. These pairs can be computed efficiently (see [6]), and it is not difficult to see that redirecting the $\varepsilon$-transitions from these pairs $(q, Z)$ is sufficient for eliminating all infinite $\varepsilon$-sequences.

We also consider the model of deterministic visibly pushdown automata (DVPA) [1]. These automata are defined with respect to a partitioned alphabet $A=A_{c} \cup A_{i} \cup A_{r}$, where $A_{c}$ contains all letters that can only occur in transitions pushing some symbol onto the stack (call symbols), $A_{r}$ those forcing the automaton to pop a symbol from the stack (return symbols), and $A_{i}$ those leaving the stack unchanged (internal symbols). Furthermore, DVPAs do not have $\varepsilon$-transitions. We also adopt the general convention that VPAs do not consider the top-most stack symbol in their transitions. This simplifies several arguments. We can make this assumption without loss of generality, because it is possible to always keep track of the top-most stack symbol in the control state.

Formally, a deterministic visibly pushdown machine over the partitioned alphabet $A=A_{c} \cup A_{i} \cup A_{r}$ is of the form $\mathscr{M}=\left(Q, A, \Gamma, \delta, q_{0}, \perp\right)$, where $\delta$ consists of three transition functions

$$
\begin{aligned}
& \delta_{c}: Q \times A_{c} \rightarrow Q \times \Gamma \\
& \delta_{r}: Q \times \Gamma \times A_{r} \rightarrow Q \\
& \delta_{i}: Q \times A_{i} \rightarrow Q
\end{aligned}
$$

Instead of defining the semantics of these transitions directly, we simply describe how the corresponding transitions in a standard DPDA would look like. A call transition $\delta_{c}(q, c)=(p, Z)$ corresponds to a set of transitions $\delta(q, Y, c)=(p, Z Y)$ for each $Y \in \Gamma_{\perp}$. A return transition $\delta_{r}(q, Z, r)=p$ corresponds to the transition $\delta_{r}(q, Z, r)=(p, \varepsilon)$, and an internal transition $\delta_{i}(q, i)=p$ to a set of transitions $\delta(q, Y, i)=(p, Y)$ for each $Y \in \Gamma_{\perp}$. Note that this definition does not admit transitions for return symbols on the empty stack. In [1] such transitions are possible, but we prefer to use the simpler model here to ease the presentation.

By adding an acceptance condition, we obtain DVPAs as in the general case. As for $\omega$-DPDAs, we are interested in $\omega$-DVPAs with Büchi or parity condition. However, we also consider a variant of the parity condition referred to as stair parity condition [11]. The condition is specified in the same way as before, however, it is evaluated only on a subsequence of the run, namely on the sequence of steps, as defined below.

A configuration $q \sigma$ in a run of a DVPA $\mathscr{A}$ is called a step if the stack height of all configurations $q^{\prime} \sigma^{\prime}$ that come later in the run is bigger than the stack height of $q \sigma$, i.e., $|\sigma| \leq\left|\sigma^{\prime}\right|$. Note that the positions of the steps do not depend on the automaton, but only on the input word, because the type of the stack operation is determined for each input symbol. We can now define stair visibly pushdown automata. The only difference to visibly pushdown automata is that they evaluate the acceptance condition only for the subsequence of the run containing consisting of the steps.

In other words, a stair parity DVPA has the same components as a parity DVPA. An input is accepted if in the run on this input the maximal priority that occurs infinitely often on a step is even. In the same way we obtain stair Büchi DVPAs, which accept if an accepting state occurs on infinitely many steps.

We end this section by introducing some more terminology for visibly pushdown automata that is used in Sections 4 and 5

The set of well matched words over $A=A_{c} \cup A_{i} \cup A_{r}$ is, intuitively speaking, the set of well-balanced words in which for each position with a call symbol there is a later position at which this call is "closed" 
by some return symbol (and vice versa, each return position has a corresponding previous call position). Formally, the set is defined inductively as follows:

- Each $a \in A_{i}$ is a well matched word.

- If $u$ and $v$ are well-matched words, then $u v$ is a well matched word.

- If $w$ is a well matched word, then $c w r$ is a well-matched word for each $c \in A_{c}$ and each $r \in A_{r}$.

The words that are created by the last rule are referred to as minimally well-matched words. Let $L_{\text {mwm }}$ denote this set, i.e., the words of the form $c w r$ with a call $c$, a return $r$, and a well-matched word $w$.

The canonical language that can be accepted by a stair Büchi DVPA but by no parity DVPA is the language $L_{\mathrm{su}}$ of strictly unbounded words, containing all words over $\langle\{c\}, \emptyset,\{r\}\rangle$ with an infinite number of unmatched calls. More formally, an infinite word is in $L_{\mathrm{su}}$ if it is of the form $w_{1} c w_{2} c w_{3} c \cdots$ for wellmatched words $w_{i}$. In [1] it is shown that $L_{\mathrm{su}}$ cannot be accepted by a parity DVPA. But it is easy to construct a stair Büchi DVPA $\mathscr{A}$ for $L_{\mathrm{su}}$ using only a single stack symbol and one accepting and one non-accepting state (see [11]), where $\mathscr{A}$ moves into the accepting state for each $c$, and into the nonaccepting state for each $r$. Note that the position after reading a $c$ is a step in the run iff this $c$ does not have a matching return. Thus, there are infinitely many unmatched calls iff there are infinitely many accepting states on steps.

\section{Regularity and Equivalence}

In this section we summarize results from [12] that show how to solve the regularity problem and the equivalence problem for weak $\omega$-DPDAs. The proof uses a reduction to the corresponding problems for DPDAs on finite words. More details on these results can be found in [12] and in [14].

The regularity problem for DPDA is the problem of deciding for a given DPDA whether it accepts a regular language. It has been shown to be decidable in [17] and the complexity has been improved in [20].

\section{Theorem 1 ([17]). The regularity problem for DPDAs is decidable.}

The rough idea of the proof is as follows. Assuming that the language of the given DPDA is regular, one shows that for each configuration above a certain height (depending on the size of the DPDA), there is an equivalent configuration of smaller height. A finite state machine can then be constructed by redirecting the transitions into higher configurations to their equivalent smaller counterparts. Here, two configurations are considered to be equivalent if they define the same language when considered as initial configuration of the DPDA. The decision method for the regularity problem is then based on the characterization of the regular languages in terms of the Myhill/Nerode equivalence. For a language $L \subseteq A^{*}$, the Myhill/Nerode equivalence is defined as follows for words $u, v \in A^{*}$ :

$$
u \sim_{L} v \text { iff } \forall w \in A^{*}: u w \in L \Leftrightarrow v w \in L .
$$

A language of finite words is regular if, and only if, it has finitely many Myhill/Nerode equivalence classes, and these classes can be used as states for a canonical finite automaton for the language.

Unfortunately, a corresponding result is not true for $\omega$-regular languages, in general. However, the subclass of weak $\omega$-regular languages possesses a similar characterization in terms of an equivalence [16]. This similarity raises the question whether the decidability results for DPDAs on finite words can be lifted to weak DPDAs on infinite words. 
In [12] it is shown that this is indeed possible. In fact, it is even possible to reduce questions for weak $\omega$-DPDAs to DPDAs on finite words. To establish such a connection, we associate a language $L_{*}(\mathscr{A})$ of finite words to a weak $\omega$-DPDA $\mathscr{A}$, which is obtained by viewing $\mathscr{A}$ as a DPDA on finite words and taking the set of states with an even priority as the set of final states.

The first attempt for reducing the regularity problem for weak $\omega$-DPDAs to the regularity problem for DPDAs would be to test $L_{*}(\mathscr{A})$ for regularity, where $\mathscr{A}$ is the given weak $\omega$-DPDA. This approach is sound because regularity of $L_{*}(\mathscr{A})$ implies $\omega$-regularity of $L_{\omega}(\mathscr{A})$ : a finite deterministic automaton for $L_{*}(\mathscr{A})$ viewed as a Büchi automaton defines $L_{\omega}(\mathscr{A})$ because it visits final states at the same positions as $\mathscr{A}$.

That the approach is not complete is illustrated by the following simple example. Consider the alphabet $\{a, b\}$ and the $\omega$-language $a^{*} b^{\omega}$ of words starting with a finite sequence of $a$ followed by an infinite sequence of $b$. Obviously, this language is regular. A weak $\omega$-DPDA $\mathscr{A}$ could proceed as follows to accept this language. It starts by pushing a symbol onto the stack for each $a$. When the first $b$ comes in the input, it changes its state and starts popping the stack symbols again. Once the bottom of the stack is reached, it changes to an accepting state and remains there as long as it reads further $b$ (if another $a$ comes, then the input is rejected). Since the finite $a$-sequence is followed by infinitely many $b$, it is guaranteed that $\mathscr{A}$ reaches the accepting state if the input is from $a^{*} b^{\omega}$. Note that this is a weak $\omega$-DPDA because it can change once from non-accepting to accepting states, and once more back to non-accepting states. The language $L_{*}(\mathscr{A})$ of this weak $\omega$-DPDA is the set of all finite words of the form $a^{m} b^{n}$ with $n \geq m$ because $\mathscr{A}$ reaches the accepting state only after it has read as many $b$ as $a$. Thus, $L_{*}(\mathscr{A})$ is non-regular although $L_{\omega}(\mathscr{A})$ is.

For this example, the problem would be solved if $\mathscr{A}$ switches to an accepting state as soon as the first $b$ is read (instead of deferring this change to the stack bottom). In general, one can show that each weak $\omega$-DPDA can be transformed in such a way that the above reduction to the regularity test for $L_{*}(\mathscr{A})$, as shown be the following theorem.

Theorem 2 ([12]). There is a normal form for weak $\omega$-DPDAs with the following properties:

1. For a weak $\omega$-DPDA $\mathscr{A}$ in normal form, the language $L_{\omega}(\mathscr{A})$ is $\omega$-regular if, and only if, $L_{*}(\mathscr{A})$ is regular.

2. Given two weak $\omega$-DPDAs $\mathscr{A}$ and $\mathscr{B}$ in normal form, $L_{\omega}(\mathscr{A})=L_{\omega}(\mathscr{B})$ if, and only if, $L_{*}(\mathscr{A})=$ $L_{*}(\mathscr{B})$.

Combining the first part of Theorem 2 with Theorem 1 , we get the decidability of the regularity problem for weak $\omega$-DPDAs.

Corollary 1 ([12]). The regularity problem for weak $\omega$-DPDAs is decidable.

The second part of the theorem can be used to show the decidability of the equivalence problem for weak $\omega$-DPDAs, based on the corresponding deep result for DPDAs.

Theorem 3 ([15]). The equivalence problem for DPDAs is decidable.

Corollary 2 ([12]). The equivalence problem for weak $\omega$-DPDAs is decidable.

The two problems for the full class of $\omega$-DPDAs remain open. In [14] a congruence for $\omega$-languages is identified that characterizes regularity within the class of $\omega$-DPDA recognizable languages (a language accepted by an $\omega$-DPDA is regular if, and only if, this congruence has finitely many equivalence classes). This might be step towards a solution for the regularity problem. However, the decidability of characterizing criterion remains open. 

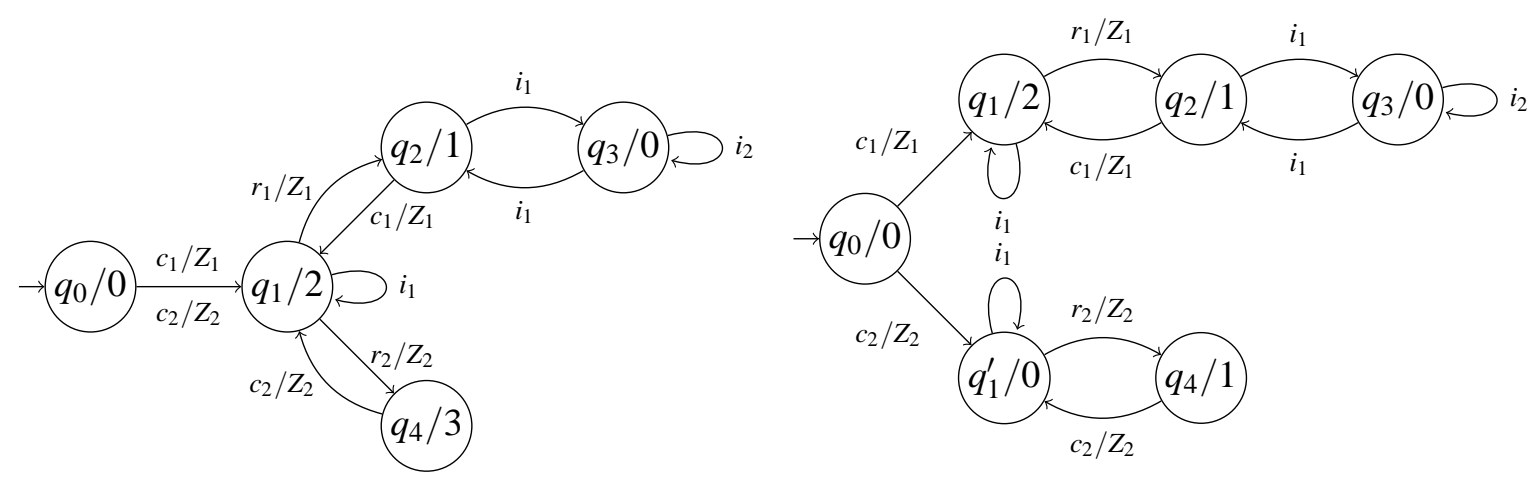

Figure 1: On the left-hand side: DVPA with minimal number of priorities for the given transition structure; on the right-hand side: equivalent DVPA with less priorities

\section{The Parity Index Problem}

In this section we are interested in the problem of reducing the number of priorities used in a parity condition. Formally, we consider the following problem. Given a parity DPDA (or stair parity DVPA) $\mathscr{A}$, compute the smallest number of priorities required for accepting $L_{\omega}(\mathscr{A})$ with a parity DPDA (or stair parity DVPA). We refer to these two variants of the problem as the parity index problem for DPDAs, and the stair parity index problem for stair parity DVPAs.

For finite parity automata, it suffices to change the priority assignment, in order to obtain an equivalent automaton with the fewest number of priorities, and this modified priority function can be computed in polynomial time [4].

For parity DPDAs the situation is different, as illustrated by the example in Figure 1 (taken from [18]). We use a DVPA in the example, where $c_{1}, c_{2}$ are calls, $r_{1}, r_{2}$ are returns, $i_{1}, i_{2}$ are internals, and $Z_{1}, Z_{2}$ are stack symbols. The transitions on call symbols are annotated with the stack symbol to be pushed, and for the return symbols with the stack symbol to be popped. The priority function of the DVPA on the left-hand side of Figure 1 (indicated as labels of the states) is minimal for the state set and the transition structure. The problem is caused by the state $q_{1}$, which is part of the loop in the upper and the lower branch. However, there is no run of the automaton that traverses both the upper and the lower branch. If the first symbol in the input is $c_{1}$, then the automaton stores $Z_{1}$ on the stack. Whenever the automaton reaches $q_{1}$ in the future, $Z_{1}$ will be on top of the stack and the automaton can only use the top branch. For the lower branch and $c_{2}$ as the first input symbol the situation is similar.

Splitting $q_{1}$ into two copies as done in the DVPA on the right-hand side of the figure, makes it possible to reassign priorities without using priority 3 .

The example illustrates that we need to take a different approach for computing the parity index of pushdown automata. This approach is also described in [14].

Let $P \subset \mathbb{N}$ be a finite set of priorities. A parity DPDA using only priorities from $P$ is referred to as a $P$-parity DPDA. To decide whether a given parity DPDA $\mathscr{A}$ has an equivalent $P$-parity DPDA, consider the following game. There are two players, referred to as Automaton and Classifier. Automaton starts in the initial configuration of $\mathscr{A}$ and plays transitions of $\mathscr{A}$. After each move of Automaton, Classifier chooses one priority from $P$. The idea is that the classifier wants to prove that there is a $P$-parity DPDA that accepts $L_{\omega}(\mathscr{A})$. If Classifier chooses priority $k$ in a move, this can be interpreted as "the parity DPDA that I have in mind would now be in a state with priority $k$ ". 
This game can be formalized as a game over a pushdown graph (basically, the configuration graph of $\mathscr{A}$ enriched by the bounded number of choices for Classifier). The winning condition states that an infinite play is won by classifier if, and only if, the two priority sequences, one induced by the configurations chosen by Automaton, the other given by the choices of Classifier, are either both accepting or both rejecting. We refer to this game as the classification game for $\mathscr{A}$ and $P$. The following result can be shown based on results for computing winning strategies in pushdown games [22].

Lemma 1. Classifier has a winning strategy in the classification game for $\mathscr{A}$ and $P$ if, and only if, there is P-parity DPDA accepting $L_{\omega}(\mathscr{A})$.

For the proof it suffices to observe the following things. If there is a $P$-parity DPDA $\mathscr{B}$ accepting $L_{\omega}(\mathscr{A})$, then Classifier can simulate the run of $\mathscr{B}$ on the inputs played by Automaton, and always choose the priority of the current state of $\mathscr{B}$. This obviously defines a winning strategy because $\mathscr{A}$ and $\mathscr{B}$ accept the same language. For the other direction one uses the fact that a winning strategy for Classifier can be implemented by a pushdown automaton that reads the moves of Automaton and outputs the moves of Classifier [22,7]. This pushdown automaton for the strategy can easily be converted into $P$-parity DPDA for $L_{\omega}(\mathscr{A})$.

For a given parity DPDA there are only finitely many sets $P$ with less priorities than $\mathscr{A}$ uses. Since it is decidable which player has a winning strategy in the classification game [22], we obtain an algorithm for solving the parity index problem for DPDAs.

Theorem 4. There is an algorithm solving the parity index problem for parity DPDAs.

\section{Stair Parity Index}

We now turn to the stair parity index problem for stair parity DVPAs. In fact, it is possible to use the same game-based approach because pushdown games with stair conditions can be solved algorithmically [11]. However, for stair parity VPAs one can also adapt the much simpler solution for computing the parity index of finite parity automata. Note that in the example from Figure 1 the "critical" state $q_{1}$ can never occur on a step (moving out of $q_{1}$ requires to read a return and thus to pop a symbol). Thus, the priority of $q_{1}$ is not important in a stair parity acceptance condition. It turns out that this is not a coincidence. The result presented below has been obtained in collaboration with Philipp Stephan, see [18].

Consider the transformation graph of a stair parity DVPA $\mathscr{A}$ defined as follows. The vertices are the states of $\mathscr{A}$. An edge from $q_{1}$ to $q_{2}$ indicates that $q_{1}$ and $q_{2}$ can occur on successive steps in a run of $\mathscr{A}$. An input connecting two successive steps of a run is either an internal symbol or a minimally wellmatched word. Therefore, this transformation graph can be computed inductively based on the definition of well-matched words from Section 2. One starts with the graph containing only the edges for the internal symbols. In each iteration one computes the transitive closure of the current graph. Denote this transitive closure by $T$. Then one checks whether there are transitions $\delta(q, c)=\left(q^{\prime}, Z\right)$ and $\delta\left(p^{\prime}, r, Z\right)=p$ for a call $c$, a return $r$, and a stack symbol $Z$, such that $\left(q^{\prime}, p^{\prime}\right) \in T$. In this case we add the edge $(q, p)$ to the graph. We repeat this procedure until no more edges are added.

The paths through the transformation graph correspond to the possible sequences of states on steps in runs of $\mathscr{A}$. We now use the algorithm from [4] to compute the minimal number of priorities required on this transformation graph, simply by viewing it as the transition graph of a finite state deterministic parity automaton. The resulting assignment of priorities is then also minimal for the stair parity DVPA $\mathscr{A}$.

Theorem 5. The stair parity index problem for stair parity DVPAs can be solved in polynomial time. 


\section{Removing the Stair Condition}

The goal is to decide for a given stair parity DVPA whether there is an equivalent parity DVPA and to construct one if it exists. We show how to decide this problem in general for stair Büchi DVPAs. We comment on the full class of stair parity DVPAs at the end of this section.

In Section 2 we described the language $L_{\mathrm{su}}$ of strictly unbounded words over $\langle\{c\}, \emptyset,\{r\}\rangle$, containing all words with an infinite number of unmatched calls. This language can be accepted by a stair Büchi DVPA but not by a parity DVPA [1]. We show that a language $L$ accepted by a stair Büchi DVPA can

- either be accepted by a parity DVPA, or

- $L$ is at least as complex as $L_{\mathrm{su}}$.

To formalize the notion of "as complex as $L_{\mathrm{su}}$ ", we need to introduce some terminology and results concerning the topological complexity of $\omega$-languages.

We can view $A^{\omega}$ as a topological space by equipping it with the Cantor topology, where the open sets are those of the form $L A^{\omega}$ for $L \subseteq A^{*}$. Starting from the open sets one defines the finite Borel hierarchy as a sequence $\Sigma_{1}, \Pi_{1}, \Sigma_{2}, \Pi_{2}, \ldots$ of classes of $\omega$-languages as follows (we omit the finite and only refer to this hierarchy as Borel hierarchy in the following):

- $\Sigma_{1}$ consists of the open sets.

- $\Pi_{i}$ consists of the complements of the languages in $\Sigma_{i}$.

- $\Sigma_{i+1}$ consists of countable unions of languages in $\Pi_{i}$.

If we denote by $B\left(\Sigma_{i}\right)$ the closure of $\Sigma_{i}$ under finite Boolean combinations, then we obtain the following relation between the classes of the Borel hierarchy, where an arrow indicates strict inclusion of the corresponding classes:

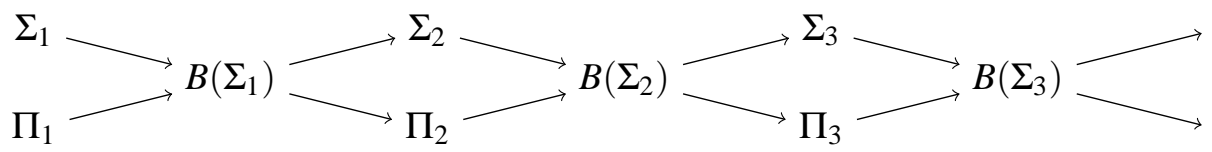

The above statement of a language $L$ being at least as complex as $L_{\mathrm{su}}$ refers to the topological complexity. It is known that languages accepted by deterministic automata (independent of the specific automaton model) with a parity condition are included in $B\left(\Sigma_{2}\right)$, and in [11] it is shown that languages accepted by stair parity DVPAS are in $B\left(\Sigma_{3}\right)$. Furthermore, it is known that $L_{\mathrm{su}}$ is a true $\Sigma_{3}$-set (it is complete for $\Sigma_{3}$ for the reduction notion introduced below) [3]. In particular, it is not contained in $B\left(\Sigma_{2}\right)$.

In our decidability proof we show that specific patterns in a stair parity DVPA induce a high topological complexity of the accepted language (namely being at least as complex as $L_{\mathrm{su}}$ ). On the other hand side, the absence of these patterns allows for the construction of an equivalent parity DVPA.

Before we introduce these patterns, we define the reducibility notion. Originally, it is defined using continuous functions. For our purposes it is easier to work with a different definition based on the Wadge game [21] (see also [3]).

Consider two alphabets $A_{1}, A_{2}$ and let $L_{1} \subseteq A_{1}^{\omega}$ and $L_{2} \subseteq A_{2}^{\omega}$. The Wadge game $W\left(L_{1}, L_{2}\right)$ is played between Players I and II as follows. In each round Player I plays an element of $A_{1}$ and Player II replies with a finite word from $A_{2}^{*}$ (the empty word is also possible). In the limit, Player I plays an infinite word $x$ over $A_{1}$, and Player II a finite or infinite word $y$ over $A_{2}$. Player II wins if $y$ is infinite and $x \in L_{1}$ iff $y \in L_{2}$.

We write $L_{1} \leq_{\mathrm{W}} L_{2}$ if Player II has a winning strategy in $W\left(L_{1}, L_{2}\right)$. The following theorem is a consequence of basic properties of $\leq \mathrm{w}$. 
Theorem 6 ([21]). If $L_{1} \leq_{\mathrm{W}} L_{2}$, then each class of the Borel hierarchy that contains $L_{2}$ also contains $L_{1}$.

We use the following consequence of Theorem 6 and the properties of $L_{\mathrm{su}}$.

Lemma 2. If $L_{\mathrm{su}} \leq_{\mathrm{W}} L$, then $L$ cannot be accepted by a parity DVPA.

Proof. As mentioned above, the languages that can be accepted by parity DPDAs are contained in $B\left(\Sigma_{2}\right)$. We sketch the proof of this folklore result for completeness: We apply Theorem 6 using the following argument. Let $\mathscr{A}$ be a parity DPDA and let $P$ be the set of priorities used by $\mathscr{A}$. Let $L_{P} \subseteq P^{\omega}$ be the sequences of priorities that satisfy the parity condition. Then $L_{\omega}(\mathscr{A}) \leq_{\mathrm{W}} L_{P}$ because in the Wadge game Player II can simply keep track of the run of $\mathscr{A}$ on the word played by Player I, and play the corresponding priorities of the states of $\mathscr{A}$. Then clearly the word played by I is in $L_{\omega}(\mathscr{A})$ iff the priority sequence of II satisfies the parity condition. Now, $L_{P}$ is easily seen to be a Boolean combination of $\Sigma_{2}$-sets.

Since $L_{\mathrm{su}}$ is not contained in $B\left(\Sigma_{2}\right)$ [3], we conclude from Theorem 6] that $L_{\mathrm{su}} \leq_{\mathrm{W}} L$ implies that $L$ cannot be accepted by a parity DVPA.

Forbidden patterns. Fix a stair Büchi DVPA $\mathscr{A}=\left(Q, A, \Gamma, q_{0}, \delta, F\right)$ and let $L=L_{\omega}(\mathscr{A})$. Recall that $L$ does not contain words with unmatched returns. We assume that all states of $\mathscr{A}$ are reachable.

For an input word $u$, states $q, q^{\prime}$, and stack contents $\sigma, \sigma^{\prime}$ we write $(q, \sigma) \stackrel{u}{\rightarrow}\left(q^{\prime}, \sigma^{\prime}\right)$ if there is a run for the input $u$ from $(q, \sigma)$ to $\left(q^{\prime}, \sigma^{\prime}\right)$. The notation $(q, \sigma) \underset{F}{\stackrel{u}{\rightarrow}}\left(q^{\prime}, \sigma^{\prime}\right)$ means that at least one state from $F$ occurs on a step in this run (for steps to be defined we assume that all prefixes of $u$ are of non-negative stack height). Dual to that we write $(q, \sigma) \underset{\notin F}{\stackrel{u}{\longrightarrow}}\left(q^{\prime}, \sigma^{\prime}\right)$ to indicate that no state from $F$ occurs on a step in this run. If we omit the input word $u$ then this means that there exists some input word.

It is not difficult to see that $L_{\mathrm{su}} \leq_{\mathrm{W}} L$ if there are words $u$ and $u^{\prime}$, a stack content $\sigma$, and a state $q \in Q \backslash F$ such that

$$
(q, \perp) \underset{F}{\stackrel{u}{\rightarrow}}(q, \sigma) \stackrel{u^{\prime}}{\rightarrow}(q, \perp)
$$

and no final state occurs on steps in this run (in a run that starts and ends in the empty stack, the steps are the configurations with empty stack). To prove $L_{\mathrm{su}} \leq_{\mathrm{W}} L$, the corresponding winning strategy for Player II in the Wadge game is: $c \mapsto u$ and $r \mapsto u^{\prime}$.

Unfortunately, the above condition is not necessary for $L_{\mathrm{su}} \leq_{\mathrm{W}} L$. Consider the stair Büchi DVPA $\mathscr{A}$ shown in Figure 2 with one call symbol $c$ and two return symbols $r_{1}, r_{2}$ (the initial state does not matter). In this automaton the simple pattern described above cannot occur because the only non-final states are $q$ and $q^{\prime}$. For these two states, words $u$ and $u^{\prime}$ as required in the pattern cannot exist for the following reasons:

- The state $q$ can only be reached via calls and therefore $(q, \perp)$ is not reachable from $(q, \perp)$.

- From $q^{\prime}$ the symbol $Z^{\prime}$ is pushed onto the stack. But $q^{\prime}$ can only be reached on popping $Z$. Therefore $\left(q^{\prime}, \perp\right)$ is not reachable from $\left(q^{\prime}, \perp\right)$.

However, the example automaton $\mathscr{A}$ contains an extended pattern that guarantees that $L_{\mathrm{su}} \leq{ }_{\mathrm{w}} L_{\omega}(\mathscr{A})$, as defined below and illustrated in Figure 3 .

Formally, we call $q, q^{\prime} \in Q \backslash F, q^{\prime \prime} \in Q, u, v, w, x, y, z \in A^{*}$, and $\sigma, \sigma^{\prime} \in \Gamma^{*}$ a forbidden pattern of $\mathscr{A}$ if $u v w x y z \in L_{\mathrm{mwm}}$ and

$$
\begin{aligned}
& (q, \perp) \underset{F}{\stackrel{u}{\rightarrow}}(q, \sigma), \quad(q, \perp) \underset{\notin F}{\stackrel{v}{\longrightarrow}}\left(q^{\prime}, \perp\right), \quad\left(q^{\prime}, \perp\right) \underset{\notin F}{\stackrel{w}{\longrightarrow}}\left(q, \sigma^{\prime}\right), \\
& (q, \perp) \stackrel{x}{\rightarrow}\left(q^{\prime \prime}, \perp\right), \quad\left(q^{\prime \prime}, \sigma^{\prime}\right) \stackrel{y}{\rightarrow}\left(q^{\prime \prime}, \perp\right), \quad\left(q^{\prime \prime}, \sigma\right) \stackrel{z}{\rightarrow}\left(q^{\prime}, \perp\right) .
\end{aligned}
$$




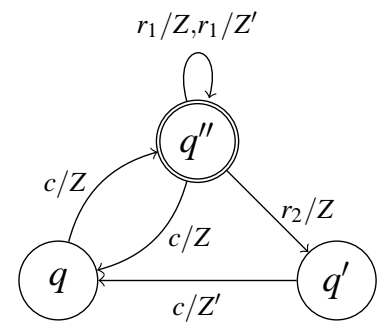

Figure 2: A stair Büchi DVPA illustrating the definition of forbidden pattern

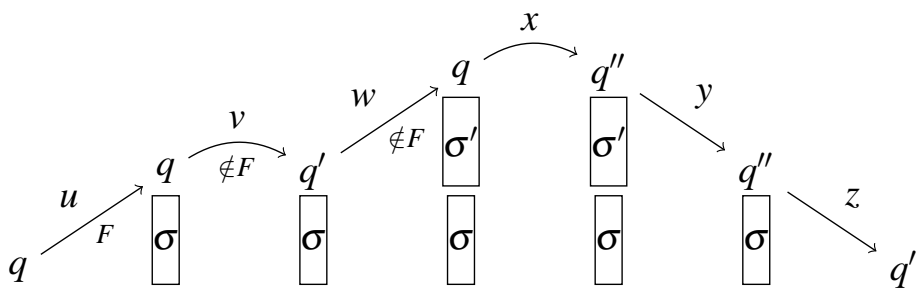

Figure 3: Forbidden pattern

Note that $\sigma^{\prime}$ might be empty. Since $q$ is a non-final state, and we require that a final state is seen on a step on the path from $q$ to $q$, the stack content $\sigma$ cannot be empty. Further note that this pattern subsumes the first simple pattern: choose $q=q^{\prime}=q^{\prime \prime}, v=w=x=y=\perp$, and $u^{\prime}=z$.

The example automaton from Figure 2 contains such a pattern for $q, q^{\prime}, q^{\prime \prime}$. the words $u=c c, v=c r_{2}$, $w=c, x=c r_{1}, y=r_{1}, z=r_{1} r_{2}$, and the stack contents $\sigma=Z Z, \sigma^{\prime}=Z^{\prime}$.

Lemma 3. If $\mathscr{A}$ has a forbidden pattern, then $L_{\mathrm{su}} \leq{ }_{\mathrm{w}} L_{\omega}(\mathscr{A})$.

Proof. We describe a winning strategy $f$ for Player II in the Wadge game. The basic idea is to play $u$ whenever Player I plays $c$, and to match the last open $u$ with $z$ whenever Player I plays $r$. However, after playing $z$, the automaton $\mathscr{A}$ is in state $q^{\prime}$ (compare Figure 3 ). Hence, to play $u$ again, we first have to play $w$ to reach $q$, producing a $\sigma^{\prime}$ on the stack. Therefore, it can happen that we first have remove these $\sigma^{\prime}$ from the stack before we can match the last open $u$ with $z$. To keep track of this, we use words over $\{0,1\}$ as memory for $f$ representing an abstraction of the stack of $\mathscr{A}(0$ corresponds to $\sigma$ and 1 corresponds to $\sigma^{\prime}$ ).

To simplify the description of $f$, we construct the moves such that $\mathscr{A}$ is always in $q^{\prime}$ after reading a finite word generated by $f$. We also assume that $q^{\prime}$ is the initial state of $\mathscr{A}$. If this is not the case, Player II can simply prepend to the first move a word leading $\mathscr{A}$ to state $q^{\prime}$.

Let $\eta \in\{0,1\}^{*}$ be the current memory content (the initial content being $\varepsilon$ ). Then the strategy $f$ works as follows:

- If Player I plays $c$, then play $w u v$ and update the memory to $01 \eta$.

- If Player I plays $r$, then let $i \geq 0$ be such that $\eta$ is of the form $1^{i} 0 \eta^{\prime}$. In this case, play $w x y y^{i} z$ and update the memory to $\eta^{\prime}$.

Let $|\eta|_{0}$ denote the number of 0 occurring in $\eta$ and let $k$ be the number of final states seen on steps in the run $(q, \perp) \stackrel{u}{\rightarrow}\left(q^{\prime}, \sigma\right)$. Note that $k \geq 1$ by definition of forbidden pattern. By induction one shows that 


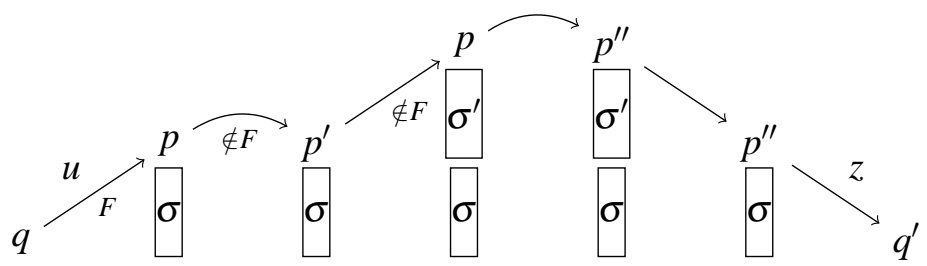

Figure 4: The relation $\left(p, p^{\prime}\right) \prec\left(q, q^{\prime}\right)$

1. after each move of Player II the number of open calls in the word played by Player I corresponds to $|\eta|_{0}$,

2. the number of final states seen on steps when $\mathscr{A}$ reads a finite word produced by $f$ is $k \cdot|\eta|_{0}$.

This implies that $\mathscr{A}$ accepts the infinite word produced by Player II according to $f$ iff the infinite word produced by Player I contains an unbounded number of unmatched calls.

Complexity of state pairs. We now show that the absence of forbidden patterns allows to construct a parity DVPA $\mathscr{A}^{\prime}$ that is equivalent to $\mathscr{A}$. In order to find an upper bound on the number of required priorities, we start by defining a measure for the complexity of pairs of non-final states. The pair $\left(q, q^{\prime}\right)$ from Figure 3 would be of infinite complexity. If we now replace the states $q$ and $q^{\prime}$ in the upper part of Figure 3 by states $p$ and $p^{\prime}$, then this indicates that the possible runs between $q$ and $q^{\prime}$ are at least as complex as those between $p$ and $p^{\prime}$. This situation is shown in Figure 4. Since $q^{\prime \prime}$ is just an auxiliary state and not of particular importance, we replaced it by $p^{\prime \prime}$ to obtain a more consistent naming scheme. We show that this relation indeed defines a strict partial order on pairs of non-final states in the case that $\mathscr{A}$ does not contain forbidden patterns.

For $p, p^{\prime}, q, q^{\prime} \in Q \backslash F$ define $\left(p, p^{\prime}\right) \prec\left(q, q^{\prime}\right)$ iff there exists $p^{\prime \prime} \in Q$ and stack contents $\sigma, \sigma^{\prime}$ such that (see Figure 4 for an illustration):

$$
\begin{array}{lll}
(q, \perp) \underset{F}{\stackrel{u}{F}}(p, \sigma), & (p, \perp) \underset{\notin F}{\longrightarrow}\left(p^{\prime}, \perp\right), & \left(p^{\prime}, \perp\right) \underset{\notin F}{\underset{\rightleftarrows}{\longrightarrow}}\left(p, \sigma^{\prime}\right), \\
(p, \perp) \rightarrow\left(p^{\prime \prime}, \perp\right), & \left(p^{\prime \prime}, \sigma^{\prime}\right) \rightarrow\left(p^{\prime \prime}, \perp\right), & \left(p^{\prime \prime}, \sigma\right) \stackrel{\rightarrow}{\rightarrow}\left(q^{\prime},\right.
\end{array}
$$

and $u z \in L_{\mathrm{mwm}}$. The words $v, w, x, y$ from the definition of forbidden pattern are not made explicit in this definition because we never need to refer to them. As for forbidden patterns, $\sigma^{\prime}$ might be empty but $\sigma$ must be non-empty.

Lemma 4. If $\mathscr{A}$ does not have a forbidden pattern, then $\prec$ is a strict partial order on pairs of states.

Proof. We have to show that $\prec$ is transitive and irreflexive (asymmetry follows from these two). The relation is obviously irreflexive because of the absence of forbidden patterns. Transitivity is illustrated in Figure 5 for $\left(r, r^{\prime}\right) \prec\left(p, p^{\prime}\right) \prec\left(q, q^{\prime}\right)$ (the stack contents are omitted). The shown pattern is obtained from $\left(r, r^{\prime}\right) \prec\left(p, p^{\prime}\right) \prec\left(q, q^{\prime}\right)$. The configurations with a frame lead to a pattern witnessing $\left(r, r^{\prime}\right) \prec\left(q, q^{\prime}\right)$.

For $\mathscr{A}$ without forbidden patterns, we assign to each pair of states a number according to its height in the partial order, i.e., $h t: Q^{2} \rightarrow \mathbb{N}$ is a mapping satisfying

$$
h t\left(q, q^{\prime}\right)=\max \left(\{0\} \cup\left\{h t\left(p, p^{\prime}\right) \mid\left(p, p^{\prime}\right) \prec\left(q, q^{\prime}\right)\right\}\right)+1 .
$$

We need the following simple observation. 


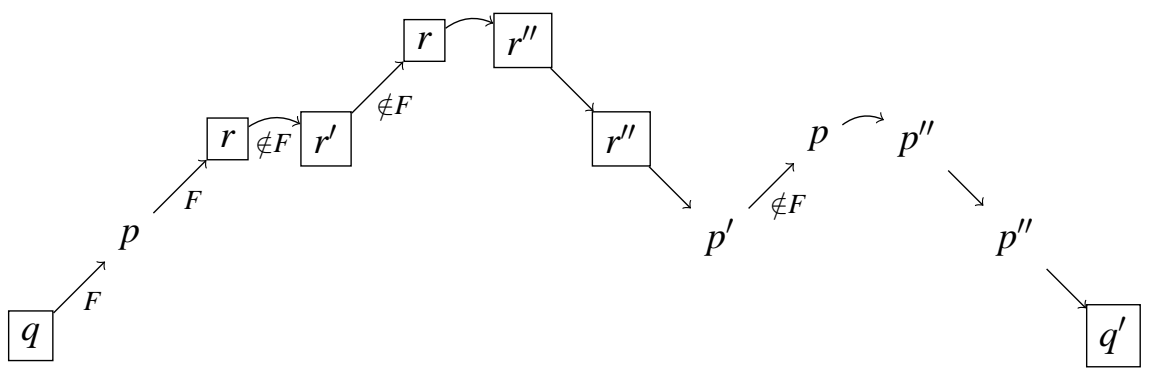

Figure 5: Transitivity of $\prec$

Lemma 5. Let $q_{1}, q_{1}^{\prime}, q_{2}, q_{2}^{\prime} \in Q \backslash F$. If there is a stack content $\sigma$ such that $\left(q_{2}, \perp\right) \stackrel{u}{\rightarrow}\left(q_{1}, \sigma\right)$ and $\left(q_{1}^{\prime}, \sigma\right) \stackrel{v}{\rightarrow}\left(q_{2}^{\prime}, \perp\right)$ with $u v \in L_{\mathrm{mwm}}$, then $h t\left(q_{2}, q_{2}^{\prime}\right) \geq h t\left(q_{1}, q_{1}^{\prime}\right)$.

Proof. The condition $\left(q_{2}, \perp\right) \stackrel{u}{\rightarrow}\left(q_{1}, \sigma\right)$ and $\left(q_{1}^{\prime}, \sigma\right) \stackrel{v}{\rightarrow}\left(q_{2}^{\prime}, \perp\right)$ with $u v \in L_{\text {mwm }}$ implies that whenever $\left(q, q^{\prime}\right) \prec\left(q_{1}, q_{1}^{\prime}\right)$, then also $\left(q, q^{\prime}\right) \prec\left(q_{2}, q_{2}^{\prime}\right)$. Thus, $h t\left(q_{2}, q_{2}^{\prime}\right) \geq h t\left(q_{1}, q_{1}^{\prime}\right)$ by definition of $h t$.

To make use of $\prec$ and $h t$ in the construction of $\mathscr{A}^{\prime}$ we need the following lemma. Note that this statement does not assume that $\mathscr{A}$ as no forbidden patterns.

Lemma 6. The relation $\prec \subseteq(Q \backslash F)^{2}$ can be computed in time polynomial in the size of $\mathscr{A}$.

Proof. In [6] it is shown that for a given configuration $p \sigma$ of $\mathscr{A}$ one can compute in polynomial time the set $\operatorname{pre}^{*}(q \sigma)$ of configurations from which there is a run to $p \sigma$, and the set $\operatorname{post}^{*}(q \sigma)$ of configurations that are reachable from $p \sigma$ by a run. These sets of configurations are sets of words over $\Gamma$, starting with a symbol from $Q$, and can be represented by finite automata.

The algorithms from [6] can be modified to consider only runs that either see a final state on a step or do not see a final state on a step, resulting in the sets $\operatorname{pre}_{F}^{*}(q \sigma), \operatorname{pre}_{\notin F}^{*}(q \sigma)$, and similarly for post.

For checking whether $\left(p, p^{\prime}\right) \prec\left(q, q^{\prime}\right)$ it is sufficient to check for each $p^{\prime \prime}$ if there are runs as required in the definition of $\prec$. This can be done by a suitable combination of the above mentioned algorithms. For example, the stack content $\sigma$ would be obtained by finding a $\sigma$ such that $p \sigma \in \operatorname{post}_{F}^{*}(q \perp)$, and $p^{\prime \prime} \sigma \in \operatorname{pre}^{*}\left(q^{\prime} \perp\right)$. Similarly for $\sigma^{\prime}$.

All these computations can be done in polynomial time, and there are only polynomially many combinations of states that have to tested.

Informal description of the parity DVPA. In a Büchi stair condition, a final state visited in a run is "erased" (in the sense that it is not considered for acceptance), if it is not on a step. If we construct a parity DVPA, then we cannot erase states like this. Instead, we use the mechanisms of different priorities to simulate erasing a state. Roughly, final states of the stair Büchi automaton are translated into even priorities. If a final state is erased, then this is compensated by visiting a higher odd priority. For the choice of the correct priorities we use the function $h t$.

In the description below, we use the terminology of " $\mathscr{A}$ closing a pair $\left(q, q^{\prime}\right)$ of states". This means that $\mathscr{A}$ was in state $q$ at some position and after reading a word $L_{\text {mwm }}$ it reached state $q^{\prime}$, i.e., $\mathscr{A}$ was in state $q$ before reading a call and reached $q^{\prime}$ after the matching return.

As mentioned above, we somehow need to determine a priority for the final states that are visited. Assume that the automaton is in configuration $(q, \beta)$ and reads a word that increases the stack height 


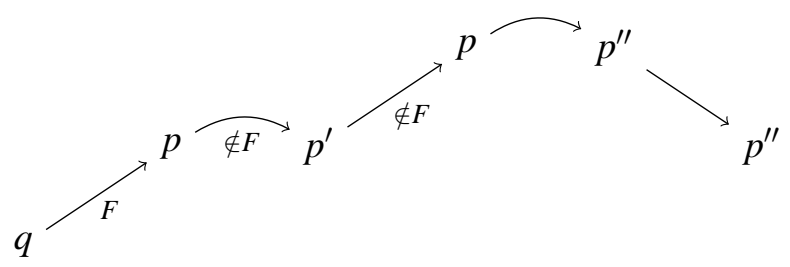

Figure 6: The pattern for determining the priority of the states with $h t\left(p, p^{\prime}\right)=i$

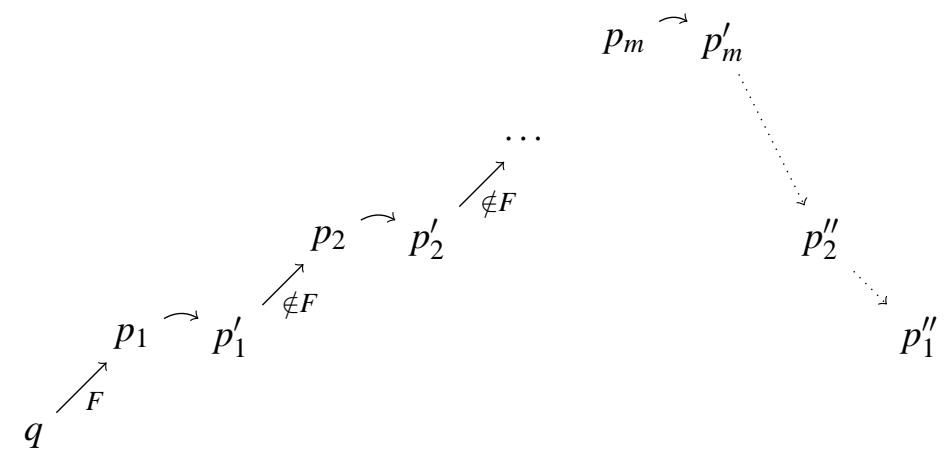

Figure 7: Detecting that each pair with $q$ is of height at least $i$.

leading to some configuration $(p, \sigma \beta)$ and visiting some final states on steps during this run. We do not know if these final states remain on steps or will be erased at some point. But if we knew, e.g., that whenever we come back to the stack content $\beta$ with, say, state $q^{\prime}$, that the pair $\left(q, q^{\prime}\right)$ is of height at least $i$, then we could signal priority $2 i$ for the final states that we have seen after $(q, \beta)$ and signal priority $2 i+1$ if we indeed close a pair $\left(q, q^{\prime}\right)$ on the level of $\beta$, and thus erasing all the final states.

Assume that we have already seen the pattern shown in Figure 6, where $\left(p, p^{\prime}\right)$ is a pair of height $i-1$. Then $h t\left(q, q^{\prime}\right) \geq i$ for every state $q^{\prime}$ that we could reach when coming back to the stack height of the configuration with $q$ at the beginning of this pattern. In particular, if $h$ is the maximal height of a pair of states, and $\left(p, p^{\prime}\right)$ are of height $h$, then we know that the final states between $q$ and $p$ cannot all be deleted because this would require closing a pair of height $h+1$.

By a simple combinatorial argument, one can see that such a pattern as shown in Figure 6 must occur if $\mathscr{A}$, before returning to the stack height of $q$, has successively closed $m:=|Q|^{3}+1$ pairs $\left(p_{1} \cdot p_{1}^{\prime}\right), \ldots,\left(p_{m} \cdot p_{m}^{\prime}\right)$ of height $i-1$ without visiting final states on steps in between, as illustrated in Figure 7 (in the picture the pairs are closed on increasing stack levels, however, they can also be on the same stack level). If we denote by $p_{i}^{\prime \prime}$ the states of $\mathscr{A}$ the next time it reaches the stack level of $\left(p_{i}, p_{i}^{\prime}\right)$ (indicated by the dotted line in the picture), then one such triple of states must occur twice, giving rise to a pattern witnessing that $h t\left(q, q^{\prime}\right) \geq i$.

To detect such situations, $\mathscr{A}^{\prime}$ maintains a counter with range from 0 to $m$ for each possible height of state pairs, and roughly behaves as follows:

- Whenever a pair of height $i$ is closed by $\mathscr{A}$, then counter $i$ is increased by one (and for technical reasons counter number 0 is increased whenever $\mathscr{A}$ visits a non-final state after reading a call or an internal symbol). To detect the closed pairs, $\mathscr{A}^{\prime}$ stores the states of $\mathscr{A}$ on the stack, and the height of state pairs can be computed by Lemma 6 
- There is an additional flag for each $i \in\{0, \ldots, h\}$ indicating whether counter number $i$ was reset because a final state of $\mathscr{A}$ has been visited (the flag is set to 1 ), or because it reached its maximal value $m$ (the flag is set to 0 ).

- When counter number $i$ reaches value $m$ (if several counters reach $m$ at the same time we take the maximal such $i$ ), then the automaton signals priority $2 i+2$ if the flag number $i$ is set, and $2 i+1$ if the flag is not set. In the next transition the counter is reset.

Formal description of the parity DVPA. Recall that $m:=|Q|^{3}+1$ and that $h$ is the maximal height of a pair of states from $Q \backslash F$.

- The states of $\mathscr{A}^{\prime}$ are of the form $(q, \chi, f)$, where $q \in Q$ is a state of $\mathscr{A}, \chi:\{0, \ldots, h\} \rightarrow\{0, \ldots, m\}$ represents the counters mentioned above, and $f:\{0, \ldots, h\} \rightarrow\{0,1\}$ represents the flag mentioned in the informal description.

- The stack symbols of $\mathscr{A}^{\prime}$ are of the form $[Z,(q, \chi, f)]$, where $Z$ is a stack symbol of $\mathscr{A}$ and $(q, \chi, f)$ is a state of $\mathscr{A}^{\prime}$.

- We now define when $\mathscr{A}^{\prime}$ can move from state $(q, \chi, f)$ to state $\left(q^{\prime}, \chi^{\prime}, f^{\prime}\right)$, depending on whether it reads a call, an internal action, or a return. In all cases, $q^{\prime}$ is the next state of $\mathscr{A}$, i.e., $\mathscr{A}^{\prime}$ simulates $\mathscr{A}$ in its first component. If $q^{\prime} \in F$, then $\chi^{\prime}=0$ and $f^{\prime}=1$, i.e., the constant functions mapping everything to 0 and 1 , respectively. The other cases for $\delta^{\prime}$ are listed below:

Call: $(q, \chi, f) \stackrel{c}{\rightarrow} \begin{gathered}\left(q^{\prime}, \chi^{\prime}, f^{\prime}\right) \\ {[Z,(q, \chi, f)]}\end{gathered}$ if $\delta(q, c)=\left(Z, q^{\prime}\right), q^{\prime} \notin F$, and

$$
\chi^{\prime}(i)=\left\{\begin{array}{l}
(\chi(i) \bmod m)+1 \text { if } i=0, \\
\chi(i) \bmod m) \text { otherwise }
\end{array} \quad f^{\prime}(i)=\left\{\begin{array}{l}
f(i) \text { if } \chi(i)<m \\
0 \text { otherwise }
\end{array}\right.\right.
$$

Internal action: $(q, \chi, f) \stackrel{a}{\rightarrow}\left(q^{\prime}, \chi^{\prime}, f^{\prime}\right)$ if $\delta(q, a)=q^{\prime}, q^{\prime} \notin F$, and $\chi^{\prime}$ and $f^{\prime}$ are as in the case of a call symbol.

$$
\begin{aligned}
& \text { Return: } \begin{array}{c}
(q, \chi, f) \\
{\left[Z,\left(q^{\prime \prime}, \chi^{\prime \prime}, f^{\prime \prime}\right)\right]}
\end{array} \stackrel{r}{\rightarrow}\left(q^{\prime}, \chi^{\prime}, f^{\prime}\right) \text { if } \delta(q, Z, r)=q^{\prime}, q^{\prime} \notin F \text {, and } \\
& \chi^{\prime}(i)= \begin{cases}\left(\chi^{\prime \prime}(i)\right. & \bmod m)+1 \text { if } q^{\prime \prime} \notin F \text { and } i \leq h t\left(q^{\prime \prime}, q^{\prime}\right), \\
\left(\chi^{\prime \prime}(i)\right. & \bmod m) \text { otherwise, }\end{cases} \\
& f^{\prime}(i)=\left\{\begin{array}{l}
f^{\prime \prime}(i) \text { if } \chi^{\prime \prime}(i)<m \\
0 \text { otherwise }
\end{array}\right.
\end{aligned}
$$

- The priority function $\Omega^{\prime}$ of $\mathscr{A}^{\prime}$ is defined as follows

$$
\Omega^{\prime}(q, \chi, f)=\left\{\begin{array}{l}
0 \text { if } \chi(i)<m \text { for all } i \\
2 d+1+f(d) \text { if } d=\max \{i \mid \chi(i)=m\}
\end{array}\right.
$$

- The initial state is $\left(q_{0}, \chi_{0}, f_{0}\right)$ with $\chi_{0}=0$ and $f_{0}=1$.

Lemma 7. The parity DVPA $\mathscr{A}^{\prime}$ is equivalent to $\mathscr{A}$.

Proof. We note the following helpful fact on reachable states $(q, \chi, f)$ of $\mathscr{A}^{\prime}$ : 
(1) If $f(i)=1$ for some $i$, then $f(j)=1$ and $\chi(i) \geq \chi(j)$ for all $j \geq i$. The initial state satisfies this property, and if we apply the definition of the transition function to a state satisfying the property, then one can easily verify that the resulting state also satisfies it.

Now consider an accepting run of $\mathscr{A}$. We show that the corresponding run of $\mathscr{A}^{\prime}$ is also accepting. Let the $k$ th state in this run of $\mathscr{A}^{\prime}$ be $\left(q_{k}, \chi_{k}, f_{k}\right)$.

If $\ell$ is a step in the run and $q_{\ell}$ is a final state of $\mathscr{A}$, then all flags are set to 1 at this point. From the definition of $\delta^{\prime}$ follows that these flags can only be set to 0 if the corresponding counter reaches value $m$ (we assume that the final state occurs on a step and therefore the run never accesses the stack symbols below). Now assume that $\mathscr{A}^{\prime}$ signals some odd priority $2 i+1$ at some position $k$ after this final state. This means that $i$ is maximal with $\chi_{k}(i)=m$, and furthermore $f_{k}(i)=0$. But if $f_{k}(i)=0$, then there must be some $k^{\prime}$ with $\ell<k^{\prime}<k$ such that $f_{k^{\prime}}(i)=1$ and $\chi_{k^{\prime}}(i)=m$ because this is the only situation in which the flag is set to 0 .

From (1) we conclude that $f_{k^{\prime}}(j)=1$ for all $j \geq i$ and hence $\Omega^{\prime}\left(q_{k^{\prime}}, \chi_{k^{\prime}}, f_{k^{\prime}}\right)$ is an even priority bigger than $2 i+1$. Thus, for each odd priority occurring after a final state on a step there is a bigger even priority also occurring after this final state. Hence, the run of $\mathscr{A}^{\prime}$ is also accepting.

For the other direction, consider a non-accepting run of $\mathscr{A}$ and as before let $\left(q_{k}, \chi_{k}, f_{k}\right)$ be the $k$ th state in the corresponding run of $\mathscr{A}^{\prime}$. There is a position such that after this position no final states of $\mathscr{A}$ occur on a step. From now on we only consider this part of the run.

Consider the sequence $k_{1}, k_{2}, k_{3}, \ldots$ of steps. As no final state occurs on a step we have the following relation between the counter values at two successive steps:

(i) If $k_{j+1}$ was reached from $k_{j}$ by reading a call or an internal symbol, then the only change of the counters is $\chi_{k_{j+1}}(0)=\left(\chi_{k_{j}}(0) \bmod m\right)+1$. The other values remain the same.

(ii) If $k_{j+1}$ was reached from $k_{j}$ by reading a minimally well-matched word, then the counters are updated as follows:

$$
\chi_{k_{j+1}}(i)= \begin{cases}\left(\chi_{k_{j}}(i)\right. & \bmod m)+1 \text { if } i \leq h t\left(q_{k_{j}}, q_{k_{j+1}}\right) \\ \left(\chi_{k_{j}}(i)\right. & \bmod m) \text { otherwise }\end{cases}
$$

The flags between two successive steps are updated as follows:

$$
f_{k_{j+1}}(i)=\left\{\begin{array}{l}
f_{k_{j}}(i) \text { if } \chi_{k_{j}}(i)<m \\
0 \text { otherwise }
\end{array}\right.
$$

Now let $d$ be the highest counter that is infinitely often increased on a step (such a counter exists because counter 0 is increased for each call and each internal symbol). Then the highest priority occurring on a step is obviously $2 d+1$ because after the first reset of counter $d$ to 0 the flag number $d$ is 0 on all following steps.

We have to show that no even priority higher than $2 d+1$ can occur infinitely often. Restrict the part of the run under consideration further to the suffix on which no counter higher than $d$ is incremented on a step. We can conclude that for successive steps connected by a minimally well-matched word we have that $h t\left(q_{k_{j}}, q_{k_{j+1}}\right) \leq d$.

We first assume that $d>0$. At the end of the proof we briefly explain the case $d=0$.

Pick $j$ such that there is $\ell$ with $k_{j}<\ell<k_{j+1}$ and $\Omega^{\prime}\left(q_{\ell}, \chi_{\ell}, f_{\ell}\right)=2 i+2$ (if no such position exists, then the run of $\mathscr{A}^{\prime}$ is clearly rejecting). For simplicity let $\left(q_{k_{j}}, \chi_{k_{j}}, f_{k_{j}}\right)=(q, \chi, f)$ and $\left(q_{k_{j+1}}, \chi_{k_{j+1}}, f_{k_{j+1}}\right)=$ $\left(q^{\prime}, \chi^{\prime}, f^{\prime}\right)$. 
We now consider the part of the run from $k_{j}$ to $\ell$ and show that $i<h t\left(q, q^{\prime}\right) \leq d$ and hence $2 i+2<$ $2 d+1$.

Since $\Omega^{\prime}\left(q_{\ell}, \chi_{\ell}, f_{\ell}\right)=2 i+2$ we know that $f_{\ell}(i)=1$ and $i$ is maximal with $\chi_{\ell}(i)=m$. If $i=0$ we know that $i<d$ by our assumption $d>0$. If $i>0$, at position $\ell$ a pair of states of height $i$ is closed. From Lemma 5] we obtain that $d \geq h t\left(q, q^{\prime}\right) \geq i$.

There are two cases to consider. If flag number $i$ was already set to 1 at position $k_{j}$, i.e., $f(i)=1$, then $i \neq d$ (as we only consider the part of the run where the flag for $d$ remains 0 forever on the steps). Together with $d \geq i$ we get $d>i$.

If $f(i)=0$, then it must be reset to 1 by visiting a final state. At the same time the counters are reset to 0 . Then $m$ pairs of height $i$ have to be closed to reach the value $\chi_{\ell}(i)=m$. Furthermore, these pairs have to closed at positions that correspond to steps in the part of the run between $k_{j}$ and $\ell$ (not steps in the whole run). Let these pairs be $\left(p_{1}, p_{1}^{\prime}\right),\left(p_{2}, p_{2}^{\prime}\right), \ldots,\left(p_{m}, p_{m}^{\prime}\right)$ (see Figure 7) and the corresponding pairs of positions be $\left(\ell_{1}, \ell_{1}^{\prime}\right) \ldots,\left(\ell_{m}, \ell_{m}^{\prime}\right)$. Now consider for each $n$ the minimal position $\ell_{n}^{\prime \prime}$ with $\ell \leq \ell_{n}^{\prime \prime} \leq k_{j+1}$ such that the stack height at $\ell_{n}^{\prime}$ and $\ell_{n}^{\prime \prime}$ is the same. Let $p_{n}^{\prime \prime}$ denote the state at the corresponding position. By the choice of $m$ we get that there are $n_{1} \neq n_{2}$ such that $\left(p_{n_{1}}, p_{n_{1}}^{\prime}, p_{n_{1}}^{\prime \prime}\right)=\left(p_{n_{2}}, p_{n_{2}}^{\prime}, p_{n_{2}}^{\prime \prime}\right)$. Denote the corresponding triple by $\left(p, p^{\prime}, p^{\prime \prime}\right)$. This triple witnesses that $h t\left(q, q^{\prime}\right)>h t\left(p, p^{\prime}\right)=i$ as illustrated in the following picture:

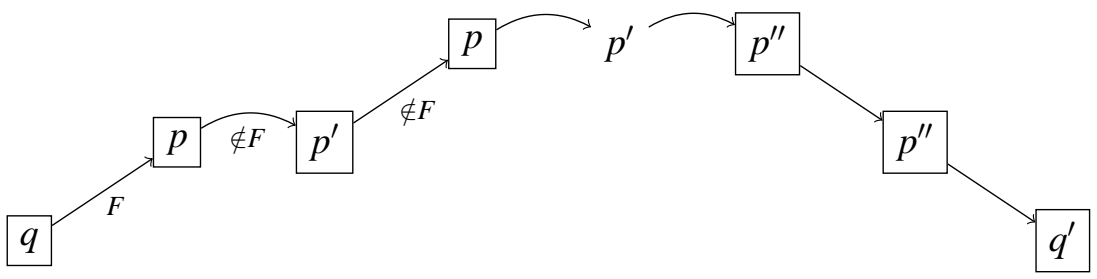

It remains to consider the case $d=0$. Consider only the suffix of the run after the position where the flag for counter 0 remains 0 on all steps and no other counter is increased on a step anymore. Then all pairs closed on steps are of height 0 and by Lemma 5 pairs closed between two successive steps are also of height 0 . So the maximal priority that we can see on this part of the run would be 2 . For this to happen, the flag for counter 0 must be 1 and counter 0 must have value $m$. The flags are only set to 1 if a final state of $\mathscr{A}$ is reached, and at the same time the counters are set to 0 . Let $q, q^{\prime}$ be the states at two successive steps, and assume that in between a final state is seen. Let $p$ be the state after the symbol following the final state. If this symbol is a call or an internal, then $(p, p) \prec\left(q, q^{\prime}\right)$ (choosing $p^{\prime \prime}=p$ ), contradicting $h t\left(q, q^{\prime}\right)=0$. Thus, each final state of $\mathscr{A}$ is immediately followed by a return. Thus, whenever the flag is set to 1 by a final state, it is immediately reset to 0 in the next transition, and thus priority 2 never occurs (on the considered part of the run).

Combining Lemmas 3 and 7 we obtain the following.

Theorem 7. A stair Büchi DVPA $\mathscr{A}$ is equivalent to a parity DVPA if, and only if, it does not contain any forbidden patterns.

The relation $\prec$ can be computed and checked for irreflexivity in polynomial time. Hence we get the following corollary.

Corollary 3. For a stair Büchi DVPA $\mathscr{A}$ it is decidable in polynomial time if it is equivalent to some parity DVPA.

A direct consequence of Lemma 7 is: 
Theorem 8. If a stair Büchi DVPA $\mathscr{A}$ is equivalent to some parity DVPA, then we can effectively construct such a parity DVPA.

It seems possible to lift the methods presented in this section to decide for general stair parity DVPAs whether the stair condition is required. We have, however, not yet worked out the details. A simpler question can be solved using the game theoretic approach for deciding the parity index problem for DPDAs: Given a stair parity DVPA $\mathscr{A}$ and a set $P$ of priorities, we can decide whether there is a parity DVPA using the priorities from $P$ that accepts $L_{\omega}(\mathscr{A})$ by using the classification game. In this case, the classification game could be formalized using a combination of a classical parity and a stair parity condition. Pushdown games with such a winning condition can be solved with the methods from [11].

\section{Conclusion}

We have considered several decidability questions for $\omega$-DPDAs. The regularity and equivalence problem are still open for the full class of $\omega$-DPDAs. We have sketched some partial results from [12] showing the decidability for these two problems for the class of weak $\omega$-DPDAs by a reduction to the corresponding problems for DPDAs on finite words. It seems that a decidability result for the full class of $\omega$-DPDAs requires new ideas.

In the second part we have analyzed the problem of simplifying the acceptance condition of $\omega$ DPDAs. We have shown that the smallest number of priorities required for accepting the language of a given parity DPDA can be computed. For the standard parity condition we have used a game approach. For stair parity DVPAs, this problem can be solved by a much simpler algorithm that uses a reduction to the computation of the parity index of a finite automaton.

We have also shown that for stair Büchi DVPAs it is decidable whether the stair condition is required or whether there exists an equivalent parity DVPA. It seems that the methods used in the proof can be generalized from stair Büchi conditions to arbitrary stair parity conditions but we have not worked out the details.

\section{References}

[1] Rajeev Alur \& Parthasarathy Madhusudan (2004): Visibly pushdown languages. In: STOC '04: Proceedings of the thirty-sixth annual ACM symposium on Theory of computing, ACM Press, New York, NY, USA, pp. 202-211, doi:10.1145/1007352.1007390

[2] Christel Baier \& Joost-Pieter Katoen (2008): Principles of Model Checking. MIT Press.

[3] T. Cachat, J. Duparc \& W. Thomas (2002): Solving Pushdown Games with a $\Sigma_{3}$ Winning Condition. In: Proceedings of the 11th Annual Conference of the European Association for Computer Science Logic, CSL 2002, Lecture Notes in Computer Science 2471, Springer, pp. 322-336, doi 10.1007/3-540-45793-322.

[4] Olivier Carton \& Ramón Maceiras (1999): Computing the Rabin Index of a Parity Automaton. ITA 33(6), pp. 495-506, doi $10.1051 /$ ita:1999129.

[5] Rina S. Cohen \& Arie Y. Gold (1978): Omega-Computations on Deterministic Pushdown Machines. JCSS 16(3), pp. 275-300, doi 10.1016/0022-0000(78)90019-3.

[6] Javier Esparza, David Hansel, Peter Rossmanith \& Stefan Schwoon (2000): Efficient Algorithms for Model Checking Pushdown Systems. In: CAV, pp. 232-247, doi 10.1007/1072216720.

[7] W. Fridman (2010): Formats of Winning Strategies for Six Types of Pushdown Games. In A. Montanari, M. Napoli \& M. Parente, editors: Proceedings of the First Symposium on Games, Automata, Logic, and 
Formal Verification, GandALF 2010, 25, Electronic Proceedings in Theoretical Computer Science, pp. 132145, doi:10.4204/EPTCS.25.14

[8] Erich Grädel, Wolfgang Thomas \& Thomas Wilke, editors (2002): Automata, Logics, and Infinite Games: A Guide to Current Research [outcome of a Dagstuhl seminar, February 2001]. Lecture Notes in Computer Science 2500, Springer, doi:10.1007/3-540-36387-4

[9] John E. Hopcroft \& Jeffrey D. Ullman (1979): Introduction to Automata Theory, Languages, and Computation. Addison Wesley.

[10] Matti Linna (1977): A Decidability Result for Deterministic omega-Context-Free Languages. Theor. Comput. Sci. 4(1), pp. 83-98, doi:10.1016/0304-3975(77)90058-5

[11] Christof Löding, Parthasarathy Madhusudan \& Oliver Serre (2004): Visibly pushdown games. In: FSTTCS 2004, Lecture Notes in Computer Science 3328, Springer, pp. 408-420, doi 10.1007/978-3-540-30538-5_34.

[12] Christof Löding \& Stefan Repke (2012): Regularity Problems for Weak Pushdown $\omega$-Automata and Games. In: Mathematical Foundations of Computer Science 2012, Lecture Notes in Computer Science 7464, Springer Berlin / Heidelberg, pp. 764-776, doi:10.1007/978-3-642-32589-2_66

[13] Dominique Perrin \& Jean-Éric Pin (2004): Infinite words. Pure and Applied Mathematics 141, Elsevier.

[14] Stefan Repke (2014): Simplification Problems for Automata and Games. Ph.D. thesis, RWTH Aachen, Germany.

[15] Géraud Sénizergues (2001): $L(A)=L(B)$ ? decidability results from complete formal systems. Theor. Comput. Sci. 251(1-2), pp. 1-166, doi $10.1016 /$ S0304-3975(00)00285-1.

[16] Ludwig Staiger (1983): Finite-State $\omega$-Languages. JCSS 27(3), pp. 434-448. Available at http://dx.doi.org/10.1016/0022-0000(83)90051-X

[17] Richard E. Stearns (1967): A Regularity Test for Pushdown Machines. Information and Control 11(3), pp. 323-340, doi:10.1016/S0019-9958(67)90591-8.

[18] Philipp Stephan (2006): Deterministic Visibly Pushdown Automata over Infinite Words. Diploma thesis, RWTH Aachen.

[19] Howard Straubing (1994): Finite Automata, Formal Logic, and Circuit Complexity. Birkhäuser, Basel, Switzerland, doi: $10.1007 / 978-1-4612-0289-9$.

[20] Leslie G. Valiant (1975): Regularity and Related Problems for Deterministic Pushdown Automata. J. ACM 22(1), pp. 1-10. Available at http://doi.acm.org/10.1145/321864.321865

[21] William W. Wadge (1984): Reducibility and Determinateness on the Baire Space. Ph.D. thesis, University of California, Berkeley.

[22] Igor Walukiewicz (2001): Pushdown Processes: Games and Model Checking. Information and Computation 164(2), pp. 234-263, doi $10.1006 /$ inco.2000.2894 